\title{
Humanidades Digitais: Novos desafios e oportunidades
}

Dália Guerreiro

CIDEHUS - Universidade de Évora

damague@fa.uevora.pt

José Luís Borbinha

Instituto Superior Técnico - INESC-ID

jlb@ist.utl.pt

\begin{abstract}
Resumo
A área das Humanidades Digitais agrega as Ciências Sociais e Humanas, as Ciências de Computação e as Ciências da Informação e Documentação. As Humanidades Digitais exibem simultaneamente, para cada disciplina, reptos próprios e grandes questões transversais. Em termos gerais, as linhas de investigação procuram o entendimento e gestão dos ciclos de vida da informação de interesse histórico e social, para reutilização como objeto de estudo pelas comunidades académicas e escolares. Os projetos existentes ou anunciados abrangem variadas áreas, destacando-se aqui aqueles que têm relação mais direta com as ciências da informação.
\end{abstract}

Palavras-chave: Humanidades digitais; Bibliotecas digitais; Biblioteconomia; Investigação em humanidades

\section{Abstract}

Digital Humanities bring together the Humanities and Social Sciences, Computer Science and Information Science. Digital humanities exhibit simultaneously, for each subject area, specifics challenges and major cross-cutting issues. In general, the lines of research seek the understanding and managing of the life cycles of information, 
with historical and social interest, in order to reuse as an object of study by academic and school communities. The existing or announced projects cover a variety of areas, from which we highlight those more concerned with information sciences.

Key-words: Digital Humanities; Digital libraries; Librarianship; Research in humanities

\section{Para a definição do conceito}

A proliferação dos media baseados na web, a disponibilização de um crescente volume de conteúdos (incluindo as redes sociais), a emergência de técnicas de análise automatizada de conteúdos e de ambientes de visualização, a computação na nuvem, etc., veio contribuir para uma revolução que nos trouxe possibilidades quase ilimitadas para a criação, a análise e a disseminação de conhecimento. As Humanidades Digitais visam ajudar a interpretar o impacto cultural e social desta nova realidade, responder às questões históricas e filológicas que daí emergem. (What Is DH?, [s.d.])

Segundo Kirschenbaum (2012) e Fitzpatrick (2012) a designação digital humanities (traduzido aqui para português como humanidades digitais) é utilizada pela primeira vez em 2004 na obra Companion to digital humanities, (Schreibman, Siemens e Unsworth, 2004), em alternativa a humanities computing, e assim evoluindo de um mero serviço para uma nova prática. (Hayles, 2012, p. 43)

Em termos genéricos, as Humanidades Digitais englobam o conjunto de pesquisas e experiências que visam facilitar a utilização dos recursos digitais no âmbito das ciências sociais e humanas, tornando-os mais intuitivos e acessíveis. Para os signatários do Manifesto', é uma «[...] transdisciplina, portadora dos métodos, dos dispositivos e das perspetivas heurísticas ligadas ao digital no domínio das ciências humanas e sociais [...]» (Manifeste des Digital humanities, 2010)2

As Humanidades Digitais, mais do que apenas conteúdos em linha, implicam uma mudança na forma de trabalhar. O objetivo das Humanidades Digitais é muito maior do que a transferência de meio, centrando-se no desafio epistemológico (Cfr. Gonçalves; Banza, 2013 , p. 5), isto é, sobre o modo como se alcança o conhecimento.

O conceito de Humanidades Digitais procura conciliar os conhecimentos e os métodos utilizados nas ciências sociais e humanas com o mundo digital. Numa primeira fase, as ações no âmbito das Humanidades Digitais centraram-se em digitalizar e disponibilizar fontes primárias, começando agora a impor-se o objetivo de construir e facultar ferramentas para a análise dessas fontes e para as expor, para que a aquisição cognitiva seja mais imediata e intuitiva. "The themes that emerged can be grouped under the following rubrics: scale, critical/productive theory, collaboration, databases, multimodal scholarship, code, and future trajectories.» (Hayles, 2012, p. 43) 
Além disso, a aposta em Humanidades Digitais também tem motivações económicas, (Kirschenbaum, 2012).

\begin{abstract}
«Desvalorizadas desde há décadas pela cultura do rendimento imediato, das falácias do "empreendedorismo", "empregabilidade" e da utilidade e consumo imediatos, as tradicionais humanidades encontram nas Humanidades Digitais um terreno bastante promissor, com impacto direto na preservação e divulgação do património, mas também na economia». (Gonçalves; Banza, 2013, p. 5)
\end{abstract}

Por isso, há que rentabilizar os investimentos realizados ao longo dos anos, tanto na digitalização massiva dos acervos, como na própria investigação. Também por esse motivo, procura ampliar-se a divulgação dessa produção científica, assumindo que se trata de fonte credível e certificada para a investigação.

\title{
Para a contextualização do conceito
}

A aplicação das Humanidades Digitais é crucial e tem sido potenciada no âmbito da educação (Greenhalgh, 2004). Em particular, as universidades com ensino de humanidades têm vindo a reconhecer a relevância deste domínio científico através da criação de departamentos de Humanidades Digitais, como o da universidade de Maryland e o University College of London.

\begin{abstract}
«Existen varios indicadores de la consolidación de un nuevo campo de estudio. Entre ellos: la formación de asociaciones, organizaciones y centros especializados, la creación de programas académicos de enseñanza, la celebración de congresos y la publicación de revistas y libros especializados. El campo de las humanidades Digitales se consolida cada vez más y existe a nivel internacional una comunidad importante que se identifica como "humanista digital». (Galina Russell, 2011, p. 4)
\end{abstract}

As associações «[...] savantes sont nombreuses dans le domaine des humanités numériques.» (Dacos; Mounier, 2014, p. 34) A Alliance of Digital Humanities Organizations, é um consórcio mundial destas organizações. O logotipo é um chapéu-de-chuva estilizado, evocando o caráter agregador da sua missão e o âmbito alargado dos seus projetos.

«The Alliance of Digital Humanities Organizations (ADHO) promotes and supports digital research and teaching across all arts and humanities disciplines, acting as a community-based advisory force, and supporting excellence in research, publication, collaboration and training» (ADHO - Alliance of Digital Humanities Organizations, [s.d.]).

As organizações que fazem parte da ADHO são também consórcios de organizações de carácter mais local, nomeadamente:

- European Association for Digital Humanities (EADH),

- Association for Computers and the Humanities $(\mathrm{ACH})$,

- Canadian Society for Digital Humanities/Société canadienne des humanités numériques $(\mathrm{CSDH} / \mathrm{SCHN})$,

- centerNet,

- Australasian Association for Digital Humanities (aaDH), 
- Japanese Association for Digital Humanites (JADH).

A ADHO permite o acesso livre à maioria das suas publicações (jornais, atas de conferências, livros, manuais, etc.) e disponibiliza igualmente informação referente aos eventos, passados e futuros, e às investigações em desenvolvimento. No entanto, o acesso ao jornal Literary and Linguistic Computing (LLC) é pago, sendo através da respetiva assinatura que as instituições ou elementos individuais se associam à ADHO.

A nível europeu, com o objetivo de fomentar a investigação e a divulgação dos projetos nesta área, foi criado o Network for Digital Methods in the Arts and Humanities (NeDiMAH), uma rede de especialistas dos vários países da União Europeia (incluindo Portugal) financiada pela European Science Foundation (ESF). Possui seis grupos de trabalho, cada um com temática específica: espaço e tempo; informação e visualização; linked data e métodos ontológicos; construção e desenvolvimento de coleções digitais para a investigação; utilização de textos em larga escala; edições digitais. Integra, ainda, três grupos transversais, cada um dos quais também com uma atribuição específica: desenvolvimento de métodos e taxonomias da Tecnologia de Informação e Comunicação (TIC); impacto da tecnologia nos métodos de investigação e nas publicações académicas; outras atividades desenvolvidas pelo NeDiMAH.

A DARIAH (Digital Research Infrastructure for the Arts and Humanities) é outra rede de organismos intergovernamentais europeus. Os países atualmente representados são: Alemanha, Áustria, Dinamarca, Eslovénia, França, Grécia, Holanda, Irlanda, Itália, Luxemburgo e Sérvia (Portugal tem em curso esforço para aderir, através da FCCN). Para garantir o financiamento a longo prazo, a rede DARIAH candidatou-se a ser reconhecida como uma estrutura ERIC (European Research Infraestruture Consortium), num processo em curso. Atualmente, a DARIAH apoia vários projetos como, por exemplo, o OpenEdition, uma plataforma para o aceso livre à informação.

Existem várias iniciativas para efetuar o levantamento dos centros de investigação, dos projetos e dos investigadores na área das Humanidades Digitais. O centerNet, ativo desde 2007, reúne cerca de 200 centros em 19 países. Outro projeto mais recente é o Atlas de Ciencias Sociales y Humanidades Digitales, ainda em construção, pelo que ainda não integra um número expressivo de investigadores e de centros.

A «construction des équipes de recherche en humanités numériques, grâce à la création de structures très particulières: les centres d'humanités numériques» (Dacos; Mounier, 2014, p. 24) é o grande motor de desenvolvimento das Humanidades Digitais.

São ainda de destacar aqui os centros de estudo dedicados às Humanidades Digitais das universidades de Harvard, de Stanford e de Maryland, nos Estados Unidos da América, e o King's College London, as universidades de Oxford e de Sheffield e o University College London, no Reino Unido. 
Existem outras organizações dignas de destaque, governamentais ou não, criadas com a missão de promover, divulgar e apoiar a investigação na área de Humanidades Digitais e fomentar a comunicação entre os intervenientes (investigadores das áreas das humanidades, da computação e da comunicação): Global Outlook::Digital Humanities (GO::DH), 4humanities, HASTAC (Humanities, Arts, Science, and Technology Alliance and Collaboratory), Red-HD (Red de humanidades Digitales de México), AHDig (Associação das Humanidades Digitais) e dh+lib. Outro caso é a Global Outlook::Digital Humanities, uma SIG (Special Interest Groups) da ADHO. "Global Outlook a pour objectif d'étendre la coopération et d'intensifier les échanges autour des humanités numériques par delà les frontières qui séparent les spécialisations mais aussi les pays et les zones linguistiques" (Dacos; Mounier, 2014, p. 35). As outras organizações, como a 4humanities e a HASTAC, defendem genericamente os mesmos propósitos. A Red-HD impulsiona as Humanidades Digitais para falantes de língua espanhola, mas centra-se no México, enquanto a AHDig dirige a sua atuação para o universo da língua portuguesa. A dh+lib foi criada pela Association of College and Research Libraries (ACRL), uma divisão da American Library Association (ALA).

A informação relativa à área de Humanidades Digitais encontra-se distribuída por monografias, atas de seminários e congressos e compilações de artigos. A literatura, tanto em periódicos como em monografias, analisa ou descreve os projetos. Porém, existe uma outra linha mais analítica, conceptual ou teórica, a qual procura equacionar os «comos» e os «porquês» do desenvolvimento na área de Humanidades Digitais.

Os primeiros artigos surgiram nos jornais científicos das áreas de linguística, computação, história e literatura. Na atualidade, existem vários periódicos da especialidade, em inglês ou francês, a maioria com revisão pelos pares (peer-review), em linha e livre acesso. Destacam-se os seguintes: o International Journal of Humanities and Arts Computing, o dhq - Digital Humanities Quarterley, o Digital Studies / Le champ numérique, o Computing in the Humanities Working Papers e o TEXT Technology: the journal of computer text processing. O Literary and Linguistic Computing apenas está disponível para os membros institucionais e individuais da ADHO ou das organizações que a ela pertencem.

\footnotetext{
«L'expérience la plus avancée et la plus intéressante du champ, à l'heure actuelle, est sans doute le projet Press Forward développé par le CHNM. II s'agit de mettre en place une chaîne complète de sélection de contenus, depuis l'agrégation de billets de blogs mis en ligne sur le web jusqu'à la publication d'une revue à comité de lecture composée d'articles issus de ces billets sélectionnés.» (Dacos; Mounier, 2014, p. 39)
}

Os resultados são colocados no Digital Humanities Now. A partir daqui, entra a seleção humana e a escolha realizada é publicada no Journal of Digital Humanities. Esta última publicação também aceita artigos que lhe são submetidos diretamente.

\section{Aplicação das Humanidades Digitais}

Constata-se que os projetos, nas várias vertentes das Humanidades Digitais, revelam ainda fragilidades em relação à persistência e preservação dos resultados, além de que, 
muitos deles, também não disponibilizam as fontes de informação utilizadas, nem referem os tratamentos aplicados para os resultados apresentados.

A representação visual de dados complexos (webmapping), que conjuga a análise do espaço e do tempo, a referenciação geográfica (GIS) e os indicadores estatísticos, constitui uma das linhas mais ativas de investigação, pelo potencial para provocar grande impacto. Estes desenvolvimentos cruzam os interesses de disciplinas como a História, a Geografia, a Estatística, as Ciências da Informação e a Computação. Um dos grupos de trabalho que segue esta linha é The Spatial History Project, da Universidade de Stanford.

A título de exemplo temos o projeto Chinese Canadian Immigrant Pipeline, 1912 1923 (Imagem 1), que pretendia facultar a visualização do fluxo migratório da China para o Canadá entre 1912 e 1923, para o que foram analisados os boletins de imigração (dados de arquivo) de cidadãos chineses nesse período de tempo. No arco cronológico de 1912 a 1923, é possível ver, por meses, a província de origem, a cidade de destino, a idade e a função dos imigrantes ao entrar no Canadá e colocar em evidência o respetivo distrito de origem, a ocupação ou o nível etário. Apesar de nem os dados originais, nem o tratamento que sofreram, estarem disponíveis, há uma descrição mínima do projeto e os tutoriais são explicativos.

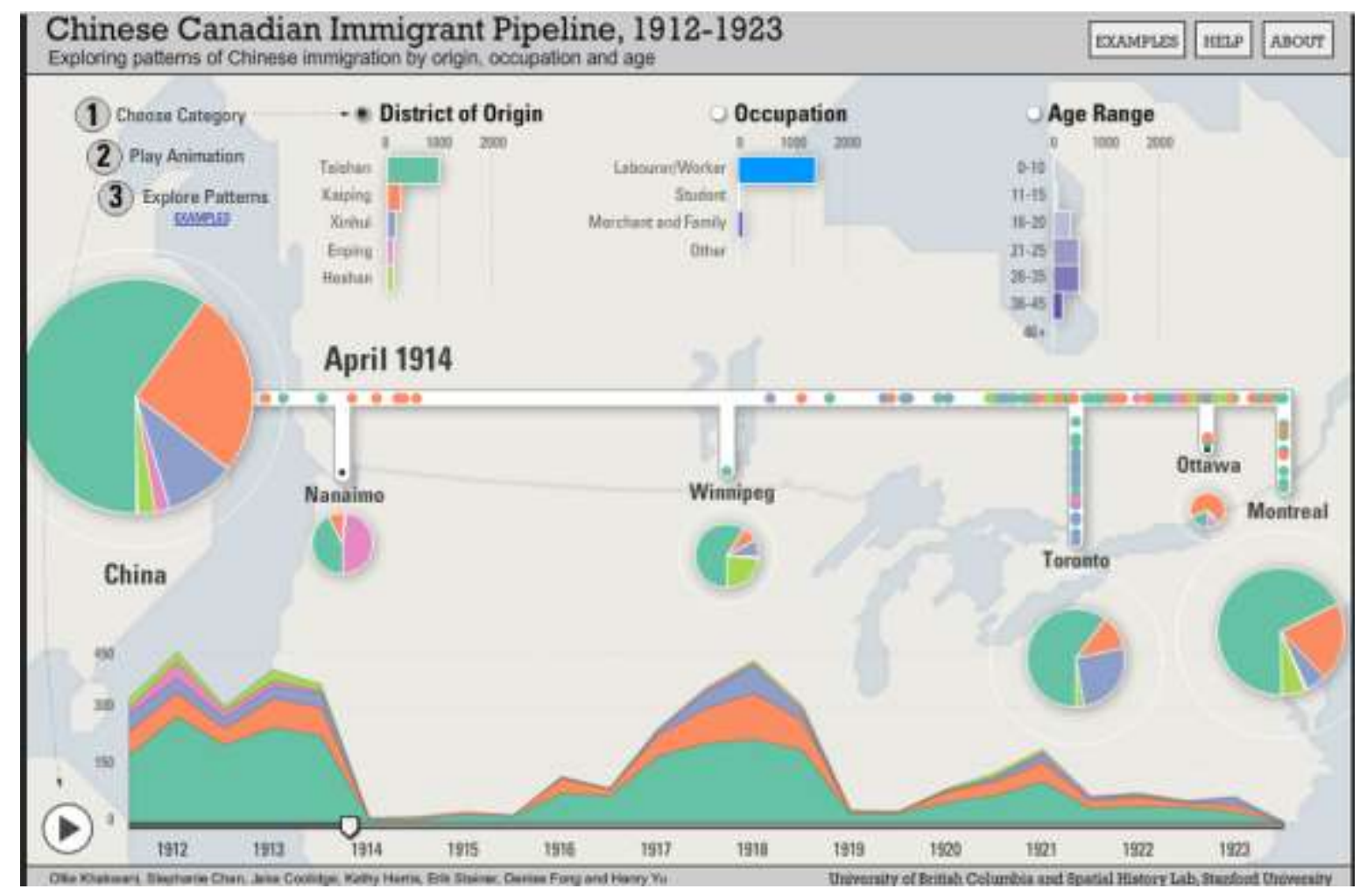

Imagem 1: Chinese Canadian Immigrant Pipeline, 1912-1923 (O estudo prévio excluiu Vancouver e Victória, pois o fluxo migratório para estas cidades foi tão intenso que, na visualização, encobria os dados referentes aos outros locais )

A tecnologia de representação em 3D constitui outra linha de investigação relevante. A construção dos modelos em 3D pode integrar imagens, dados geográficos, gráficos, 
tabelas, referências a material de arquivo ou biblioteca, etc. A respetiva visualização pode ser feita para permitir uma análise descritiva e analítica dos locais.

Um projeto português que utiliza a tecnologia 3D é $L X$ Conventos - Da cidade sacra à cidade laica $\mathrm{O}$ produto final irá consistir num mapa 3D da cidade de Lisboa. Este projeto pretende facultar informação sobre os conventos e sua envolvente. Para tal, é necessário estudar, de forma sistemática e integrada, o impacto da extinção das ordens religiosas no desenvolvimento, funções e imagens da nova cidade liberal e secular. O projeto iniciado em 2013 utiliza, como fontes de informação, mapas antigos e atuais, fotografias, maquetes, etc. A integração de todas as informações é realizada através do reconhecimento automático dos monumentos, sendo necessário a validação humana.

Outra linha de investigação incide sobre a construção de bibliotecas digitais vocacionadas para a investigação, onde se inclui a utilização e a reutilização da informação para a criação de novo conhecimento. Esta linha de investigação aborda principalmente a descrição e a estruturação do objeto digital, incluindo a conservação e a preservação da informação ao longo do tempo.

Neste âmbito e na vertente da linguística, pode citar-se, entre outros, o projeto Perseus Digital Library, constituindo um exemplo de projeto colaborativo (Guerreiro; Calixto; Borbinha, 2012).

A adequação das bibliotecas digitais às novas exigências está a criar um novo dinamismo na investigação, referindo-se, como exemplo, o protótipo para disponibilizar jornais em linha (Imagem 2). Este projeto, realizado com fundos comunitários do programa CIP 2007 - 2013, é acessível através da Europeana and The European Library e pretende disponibilizar cerca de 10 milhões de páginas de jornal. O objetivo é disponibilizar os jornais de forma mais acessível, permitindo uma melhor recuperação da informação.

Para tal, o protótipo apresenta:

- Pesquisa em texto integral;

- A pesquisa por data, sendo disponibilizado um calendário que vai de 1813 a 2012;

- As folhas de rosto dos jornais publicados: a página exibida é a página da primeira edição, mas há a hipótese de se escolher o ano;

- Possibilidade de navegação pelo título do jornal.

Uma vez realizada a seleção do jornal e da página a consultar, são apresentados, em simultâneo, o texto resultante do OCR da página e respetiva imagem, com indicação da palavra ou expressão de consulta. 


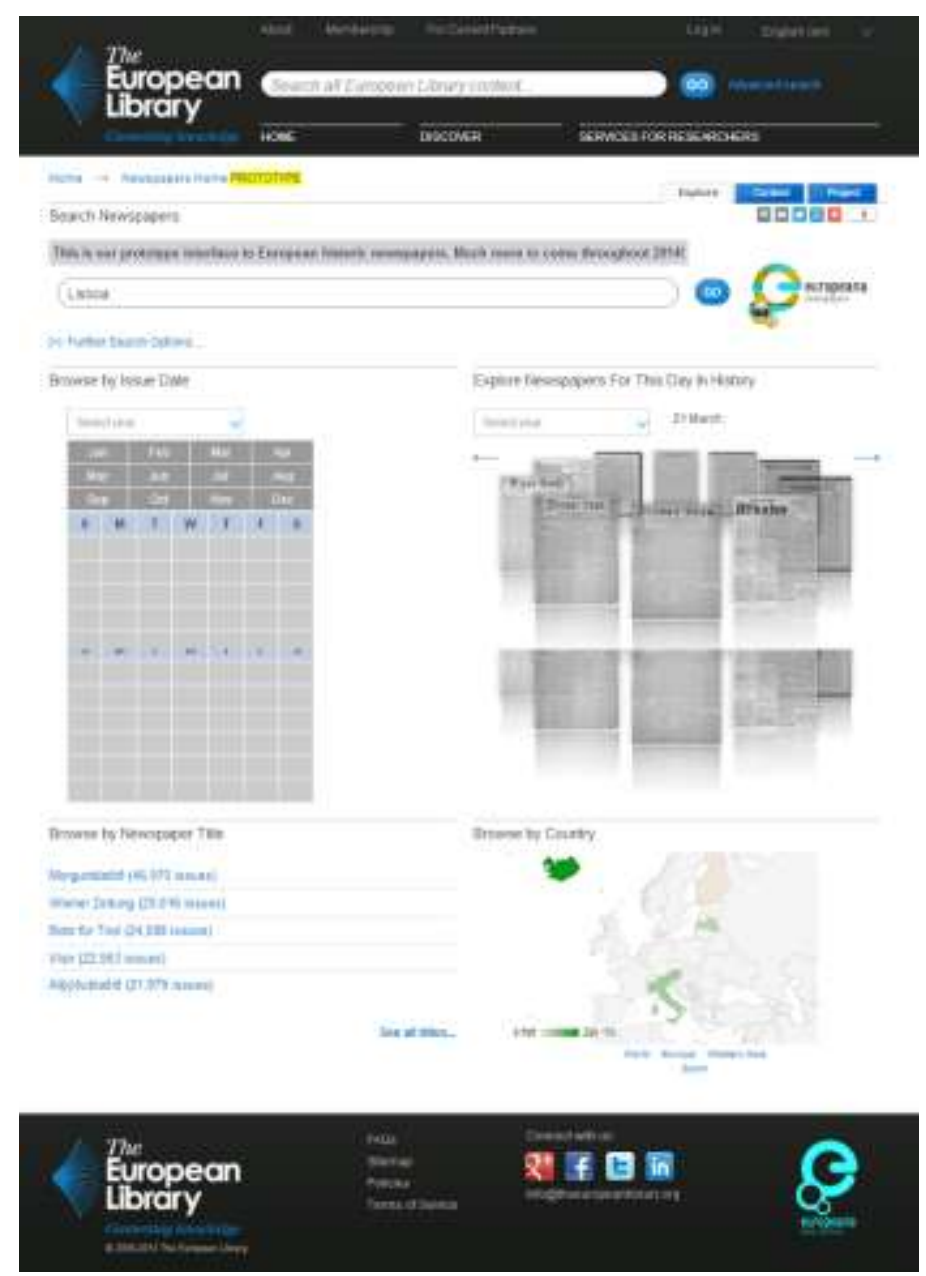

Imagem 2: - Newspapers Home PROTOTYPE, página inicial

Outro exemplo interessante é ainda o projeto Bibliothèques Virtuelles Humanistes (Imagem 3), criado em 2002 pelo Centre d'Études Supérieures de la Renaissance - CERS com a colaboração do Institut de recherche et d'histoire des textes - IRHT secção de humanismo, que disponibiliza, em linha, uma biblioteca digital coerente com o fac-simile de obras renascentistas digitalizadas pelos parceiros do projeto.

Pode aceder-se às obras através de pesquisa simples (título, autor, data, edição, cota) ou avançada (os vários campos da ficha bibliográfica). Também está disponível uma lista por autor ou título, sendo possível fazer a ordenação por autor, título e data. O acesso à obra digitalizada é feito clicando na obra. Existe a hipótese de se consultar o sumário da obra (abre em janela aparte) ou o thumbnail das imagens. Quando se navega na obra, mantém-se a referência à página em que nos encontramos, mas a indicação do capítulo apenas aparece na página em que inicia. O zoom abre a imagem numa outra janela, não havendo a hipótese de se navegar nas obras com as imagens aumentadas. 


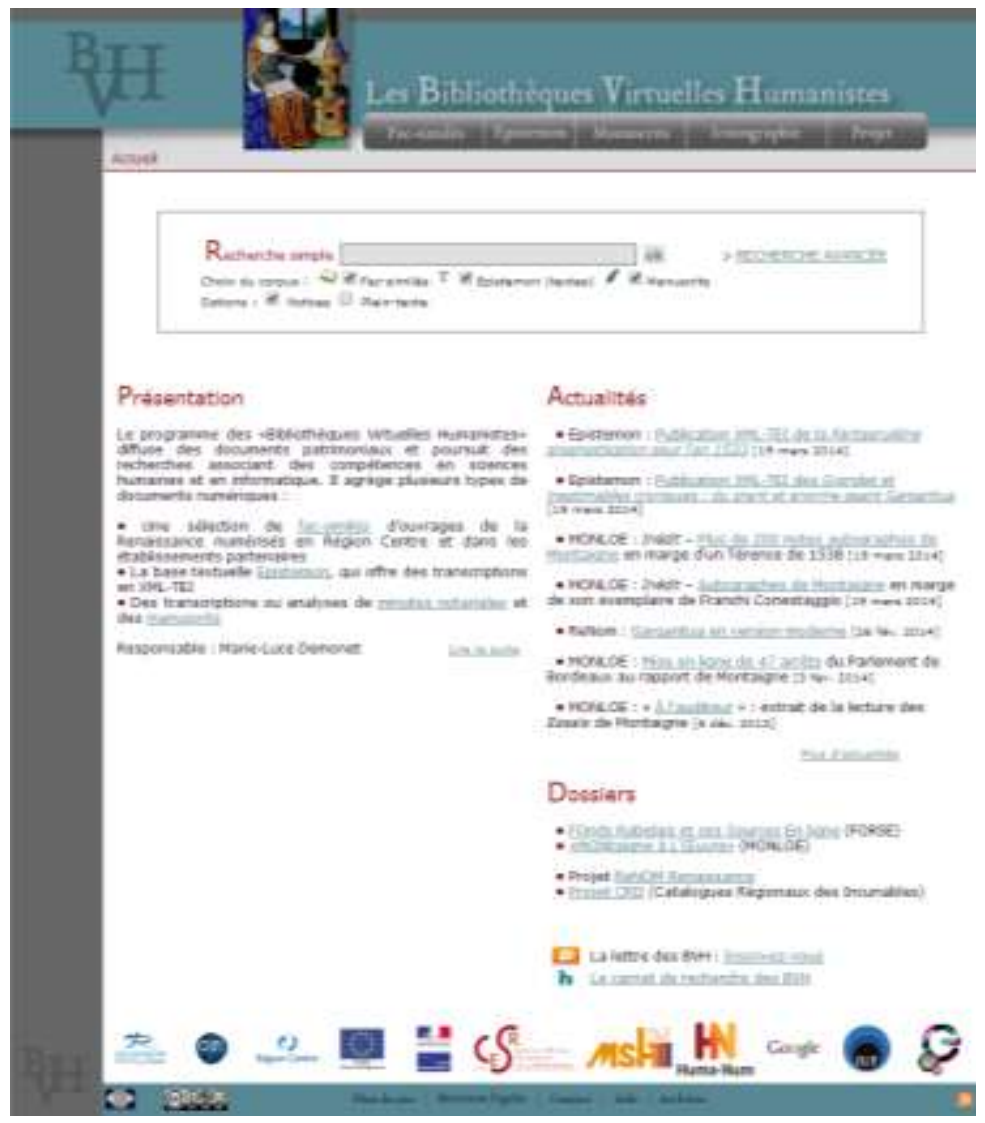

Imagem 3: Bibliothèques Virtuelles Humanistes, página inicial

No Epistemon - Corpus de textos da Renascença (Imagem 4), base de dados textual com transcrição dos documentos para XML-TEI, a pesquisa faz-se por palavra no corpo do texto ou através dos elementos da ficha bibliográfica. A pesquisa cruzada entre os elementos da ficha bibliográfica e a palavra a pesquisar conduz à página, à secção ou ao parágrafo em que a pesquisa se enquadra. As regras utilizadas na transcrição estão disponíveis em linha. Também é possível aceder à obra e ver, em simultâneo, o fac-simile e a respetiva versão textual.

$\mathrm{Na}$ secção de iconografia, é disponibilizada a pesquisa através do Iconclass. As imagens são complementadas com a informação relativa à obra onde se encontra. Estão também disponíveis bases de dados com as letras capitulares, com as marcas dos impressores, e com os retratos, sendo sempre feita a referência à obra de origem.

Atualmente, na secção dos manuscritos, procede-se à transcrição, codificada em TEl, de documentos dos séculos XV e XVI.

As edições digitais escolares também constituem outra linha de investigação, assumindo como objetivos a definição do atual estado da edição digital e a elaboração de um conjunto de boas práticas para a preservação da informação no tempo e a respetiva interoperabilidade. 


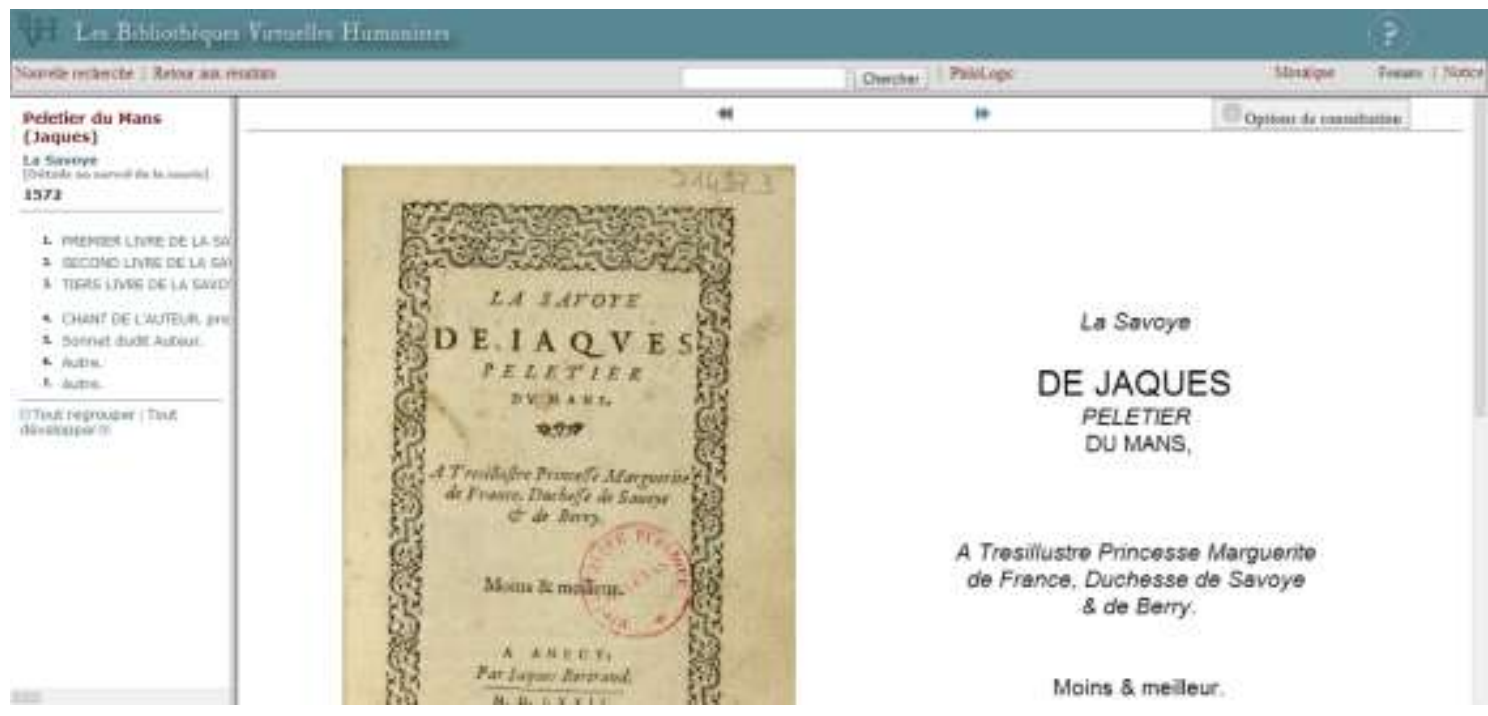

Imagem 4: - Bibliothèques Virtuelles Humanistes, acesso à obra no Epistemon

A OpenEdition, desenvolvida pelo Centre pour l'édition électronique ouverte - CLÉO, unidade que congrega o CNRS, o EHESS, a Universidade Aix-Marseille e a Universidade de Avignon, oferece à comunidade académica uma plataforma internacional de publicação e divulgação da informação em ciências sociais e humanas. É constituída pelas seguintes plataformas: Hypotheses, que alberga blogues denominados cadernos de investigação; Revues, para os periódicos; Calenda, com a agenda e a divulgação de eventos; e os ebook's.

Os blogues que integram a Hypotheses são pré-avaliados por uma comissão académica e podem receber um ISSN (International Standard Serial Number), código de registro internacional das publicações periódicas. A atribuição de ISSN a blogues, reconhecendo-os como publicações científicas, é inédita.

A Revues é um portal de revistas científicas em linha no domínio das humanidades e das ciências sociais. Privilegia a disponibilização de artigos em texto integral e em acesso livre. As revistas são selecionadas por um conselho científico. Cada revista tem uma formatação individualizada, mas na página de acolhimento de cada uma, além da pesquisa por palavra, é dado destaque ao último número e apresentam-se os índices por autor (ordem direta) e por palavra-chave, o histórico da revista, as informações, etc. Em cada artigo pode consultar-se: o resumo; o índice (palavras-chave); o mapa (sumário); o texto; a bibliografia; os anexos; as notas; as ilustrações; citação (como citar o artigo); e os dados biográficos do autor. O texto está disponível em PDF e em HTML. Os parágrafos estão numerados, o que, por se manterem independentemente do tamanho do ecrã em que o artigo é visualizado, facilita a referenciação. 


\section{Conclusão}

É possível afirmar-se que os «technological advances have altered the way that researchers capture, curate, analyze and visualize data at every scale». (Collins; Jubb, 2012). A área das Humanidades Digitais deve ser pensada como uma nova forma de solucionar os problemas da investigação em humanidades, mas preservando a tradição, dando atenção à complexidade e mantendo as modalidades de análise em profundidade, crítica e interpretação (Evans; Rees, 2012, p. 29).

A área de Humanidades Digitais é no entanto ainda encarada como uma «caixa negra» (Rieder; Rohle, 2012), da qual tudo pode sair, sem que se perceba exatamente como foi produzido. Há, por isso, que criar conteúdos apelativos para o ensino e para o grande público. Para os investigadores, torna-se imperioso que, em simultâneo, sejam disponibilizadas as fontes utilizadas e se refiram as leituras e as metodologias efetuadas.

A utilização e a reutilização da informação também levantam questões sobre o direito de autor e o copyright (quem é o autor?) não só para a comunidade dos humanistas digitais, mas para todos os utilizadores, o que implica prudência na implementação de novos modelos. No domínio específico das bibliotecas digitais, começa a ser óbvia a necessidade de ir além do fac-símile em PDF, dado que este formato, apesar de poder incluir o OCR das obras, não permite a estruturação do texto com as mesmas possibilidades do TEI.

As Humanidades Digitais afirmam-se como um campo fértil da investigação, mas o principal desafio é ainda a definição de modelos genéricos para a sistematização e partilha (reuso) da informação reunida, o que faz com que de momento cada projeto seja ainda como que uma ilha.

\section{Referências bibliográficas}

ADHO - Alliance of Digital Humanities Organizations - [Em linha] [Consult. 4 mar. 2014]. Disponível em WWW:<URL:http://adho.org/>.

COLLINS, Ellen; JUBB, Michael (2012) - How do researchers in the Humanities use information resources ? Liber Quarterly. ISSN 2213-056X. 21:2, 176-187.

DACOS, Marin; MOUNIER, Pierre (2014) - Humanités numériques: État des lieux et positionnement de la recherche française dans le contexte international [Em linha]. Paris : Institut Français. Disponível em WWW:<URL:http://www.institutfrancais.com/fr/actualit\%C3\%A9s/humanitesnumeriques>. ISBN 9782354761080.

EVANS, Lieghton; REES, Sian (2012) - An interpretation of digital humanities. In BERRY, DAVID M. (Ed.) - Understanding digital humanities. Houndmills, Basingstoke, Hampshire : Palgrave Macmillan, 2012. ISBN 9780230371934. p. 21-41. 
FITZPATRICK, Kathleen (2012) - The humanities, done digitally. In GOLD, MATTHEW K. (Ed.) Debates in the Digital Humanities [Em linha]. Minneapoli ed. [S.I.] : University of Minnesota Press [Consult. 23 feb. 2014]. Disponível em WWW:<URL:http://dhdebates.gc.cuny.edu/debates/text/30>. ISBN 978-0816677955. p. 504.

GALINA RUSSELL, Isabel (2011) - ¿Qué son las Humanidades Digitales? Revista Digital Universitaria. ISSN 1607-6079. 12:7

GONÇALVES, Maria Filomena; BANZA, Ana Paula (2013) - In limine. In GONÇALVES, MARIA FILOMENA; BANZA, ANA PAULA (Eds.) - Património textual e Humanidades Digitais: da antiga à nova Filologia [Em linha]. Évora : CIDEHUS, 2013 Disponível em WWW:<URL:http://dspace.uevora.pt/rdpc/handle/10174/10468>. ISBN 978-98995669-7-2

GREENHALGH, Michael (2004) - Art History. In SCHREIBMAN, Susan; SIEMENS, Raymond George; UNSWORTH, John (Eds.) (2004) - A companion to Digital Humanities [Em linha]. Malden, Oxford : MA, Blackwel [Consult. 20 sep. 2012]. Disponível em WWW:<URL:http://www.digitalhumanities.org/companion/>. p. 42-.

GUERREIRO, Dália Maria; CALIXTO, José António; BORBINHA, José (2012) - Bibliotecas Digitais para as Humanidades: Novos desafios e oportunidades. In Actas do Congresso Nacional de Bibliotecários, Arquivistas e Documentalistas [Em linha]. Lisboa - Fundação Calouste Gulbenkian, 18, 19 e 20 de Outubro de 2012 : BAD - Associação Portuguesa de Bibliotecários, Arquivistas e Documentalistas, 2012 [Consult. 24 apr. 2014]. Disponível em WWW:<URL:http://www.bad.pt/publicacoes/index.php/congressosbad/article/view/3 $54 / p d f>$.

HAYLES, N. Katarine (2012) - How we think: transforming power and digital technologies. In BERRY, DAVID M. (Ed.) - Understanding digital humanities. Houndmills, Basingstoke, Hampshire : Palgrave Macmillan. ISBN 9780230371934

KIRSCHENBAUM, Matthew G. (2012) - What is digital humanities and what's it doing in english departments. In GOLD, MATTHEW K. (Ed.) - Debates in the Digital Humanities [Em linha]. Minneapolis : University of Minnesota Press. Disponível em WWW:<URL:http://dhdebates.gc.cuny.edu/debates/text/38>. ISBN 9780816677948. p. 516.

MANIFESTE DES DIGITAL HUMANITIES - [Em linha]. Paris : [s.n.] [Consult. 1 jul. 2012]. Disponível em WWW:<URL:http://tcp.hypotheses.org/497>.

RIEDER, Bernhard; ROHLE, Theo - Digital methods: five challenges. In BERRY, DAVID M. (Ed.) Understanding digital humanities. Houndmills, Basingstoke, Hampshire : Palgrave Macmillan, 2012. ISBN 9780230371934. p. 64-.

SCHREIBMAN, Susan; SIEMENS, Ray; UNSWORTH, John (EDS.) (2004) - A companion to digital humanities [Em linha]. Oxford : Blackwell, 611 p. [Consult. 12 apr. 2012]. Disponível em WWW:<URL:http://www.digitalhumanities.org/companion/>. ISBN 9781405103213.

WHAT IS DH? - [Em linha] [Consult. 14 feb. 2014]. Disponível em WWW:<URL:http://cdh.ucla.edu/about/what-is.html>. 


\section{Agradecimentos}

Esta investigação é financiada pela FCT através da bolsa de formação avançada, SFRH / BD / 82229 / 2011.
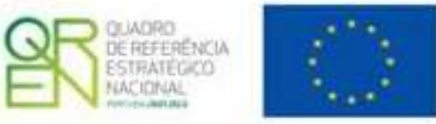

UNLĀO EUROPEIA

Fundo Europes

(9)

FCT 


\section{Anexo}

\section{Lista de endereços eletrónicos}

\section{4humanities}

http://4humanities.org/

Alliance of Digital Humanities Organizations (ADHO)

http://adho.org/

American Library Association (ALA)

http://www.ala.org/

Associação das Humanidades Digitais (AHDig)

http://ahdig.org/

Association for Computers and the Humanities (ACH)

http://www.ach.org/

Association of College \& Research Libraries (ACRL)

http://www.ala.org/acrl/

Australasian Association for Digital Humanities (aaDH)

http://aa-dh.org/

Bibliothèques Virtuelles Humanistes

http://www.bvh.univ-tours.fr/

Bibliothèques Virtuelles Humanistes - Epistom

http://www.bvh.univ-tours.fr/Epistemon/index.asp

Canadian Society for Digital Humanities / Société canadienne des humanités numériques $(\mathrm{CSDH} / \mathrm{SCHN})$

http://csdh-schn.org/

centerNet

http://digitalhumanities.org/centernet/

Centre d'Études Supérieures de la Renaissance

http://cesr.univ-tours.fr/

Centre pour l'édition électronique ouverte (CLEO)

http://cleo.openedition.org/

Chinese Canadian Immigrant Pipeline, 1912-1923

http://www.stanford.edu/group/spatialhistory/cgi-bin/site/viz.php?id=393

Computing in the Humanities Working Papers

http://projects.chass.utoronto.ca/chwp/

$\mathrm{dh}+\mathrm{lib}$

http://acrl.ala.org/dh/

Digital Humanities at Harvard 
http://isites.harvard.edu/icb/icb.do?keyword=k15573

Digital Humanities Now

http://digitalhumanitiesnow.org/

Digital Humanities Quarterly (DHQ)

http://www.digitalhumanities.org/dhq/

Digital Research Infrastructure for the Arts and Humanities (DARIAH)

http://www.dariah.eu/

Digital Studies / Le champ numérique

http://www.digitalstudies.org/

Digital.Humanities@Oxford

http://digital.humanities.ox.ac.uk/

Directorio de centros de investigación

http://grinugr.org/centros/

European Association for Digital Humanities (EADH)

http://eadh.org/

European Library, ewspapers Home PROTOTYPE

http://www.theeuropeanlibrary.org/tel4/newspapers

European Science Foundation (ESF)

http://www.esf.org/

Global Outlook::Digital Humanities

http://www.globaloutlookdh.org/

Humanidades Digitales RedHD

http://www.humanidadesdigitales.net/

Humanities Research Institute, Sheffield

http://www.sheffield.ac.uk/hri/technology

Humanities, Arts, Science and Technology Alliance and Collaboratory (HASTAC)

http://www.hastac.org/

Hypotheses

http://hypotheses.org/

Iconclass

http://www.iconclass.org/

Institut de recherche et d'histoire des textes (IRHT)

http://www.irht.cnrs.fr/

International Journal of Humanities and Arts Computing

http://www.euppublishing.com/loi/ijhac

Japanese Association for Digital Humanities (JADH)

http://www.jadh.org/

Journal of Digital Humanities (JDH) 
$\underline{\text { http://journalofdigitalhumanities.org/ }}$

Literary and Linguistic Computing (LLC)

http://llc.oxfordjournals.org/

LX Conventos - Da cidade sacra à cidade laica

http://citi.di.fct.unl.pt/project/project.php?id=108

Maryland Institute for Technology in the Humanities

http://mith.umd.edu/

Network for Digital Methods in the Arts and Humanities (NeDiMAH)

http://www.nedimah.eu/

OpenEdition

http://www.openedition.org/

Perseus digital library

http://www.perseus.tufts.edu/

\section{Revus}

http://www.revues.org/

School of Arts \& Humanities, Digital Humanities, King's College de Londres

http://www.kcl.ac.uk/artshums/depts/ddh/index.aspx

Spatial History Project at Stanford University

http://www.stanford.edu/group/spatialhistory

Stanford Humanities Center

http://shc.stanford.edu/digital-humanities

TEXT Technology

http://texttechnology.mcmaster.ca/

UCL Centre for Digital Humanities, University College London

https://www.ucl.ac.uk/dh/

\section{Notas}

${ }^{1}$ O manifesto surge na sequência do ThatCamp 2010 em Paris, uma não conferência sobre o digital humanities. Atualmente os signatários, investigadores maioritariamente europeus e respetivas instituições, somavam, até final de 2011, 293 individuais e 10 instituições. Atualmente (03-03-2014), encontram-se registados 3.155 pessoas.

${ }^{2}$ A versão original é em francês, mas encontra-se traduzida no sitio eletrónico do ThatCamp Paris em http://tcp.hypotheses.org/497 